\title{
Atrofia muscular espinal: Caracterización clínica, electrofisiológica y molecular de 26 pacientes
}

\author{
CLAUDIA CASTIGLIONI ${ }^{1,2}$, JORGE LEVICÁN $^{3}$, ELIANA RODILLO $^{1}$, \\ MARÍA ANGÉLICA GARMENDIA ${ }^{3}$, ALEJANDRA DÍAZ ${ }^{2}$, \\ LORENA PIZARRO ${ }^{1,4}$, LUIS CONTRERAS ${ }^{3}$
}

\section{Clinical, electrophysiological and molecular study of 26 chilean patients with spinal muscular atrophy}

Background: Spinal Muscular Atrophy (SMA) is an autosomal recessive disorder affecting the anterior horn cells of the spinal cord resulting in muscle weakness and atrophy, linked to the homozygous disruption of the survival motor neuron 1 (SMN1) gene. It is the leading genetic cause of infant death. It has been classified into three types based on the severity of symptoms. Type I SMA is the most severe form with death within the first 2 years of life. Type II and III SMA patients show intermediate and mild forms of the disorder. Aim: To describe the clinical and electrophysiological findings of 26 Chilean patients with SMA with molecular confirmation. Patients and Methods: Retrospective multicenter analysis of patients with SMA assessed between 2003 and 2010. The diagnosis was suspected on clinical and electrophysiological criteria. Since 2006 molecular genetics confirmation was implemented in one of our centers. Results: Twenty-six patients between 2 months and 18 years of age at presentation were analyzed; 15 (58\%) were males. SMA I, II and III clinical criteria were observed in 4 (15.4\%), 11(42.3\%) and 11(42.3\%) patients, respectively. All had proximal muscle weakness and atrophy. Electromyography showed features of acute denervation or re-innervation with normal motor and sensory nerve conduction. Nine patients required a muscle biopsy. The genetic confirmation of the disease by PCR technique followed by restriction fragment length polymorphism method disclosed the SMN1 gene deletion in all 26 cases. All patients died secondary to respiratory failure, between eight and 14 months of life. Conclusions: An adequate clinical and molecular diagnosis of spinal muscular atrophy will help for a better management of these patients.

(Rev Med Chile 2011; 139: 197-204).

Key words: Electrophysiology; Genetic testing; Spinal muscular atrophies of childhood.

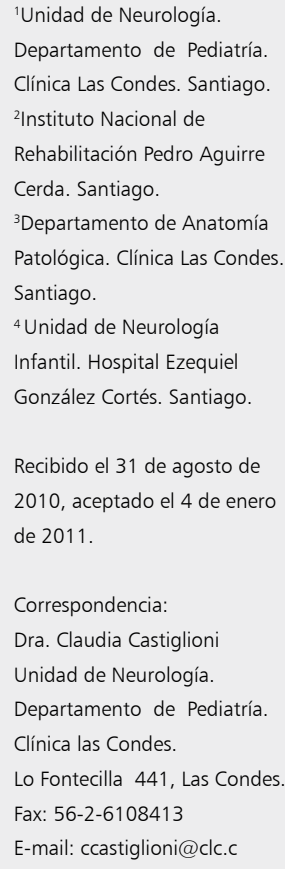

I a atrofia muscular espinal (AME) es una enfermedad neurodegenerativa que afecta la médula de enfermedad autosómica recesiva después de la fibrosis quística ${ }^{1}$. Es la causa genética más fre- cuente de mortalidad en lactantes ${ }^{1,2}$, con una incidencia mundial descrita entre $1 / 6.000$ y $1 / 10.000$ nacimientos y una tasa de portadores entre $1 / 35$ y $1 / 50^{1-3}$. En nuestro país no existen datos de su prevalencia.

Clínicamente se caracteriza por una debilidad 
y atrofia muscular generalizada de predominio proximal, que comienza en extremidades inferiores, extendiéndose a tronco y extremidades superiores en grado variable según el tipo clínico ${ }^{2,3}$. La capacidad cognitiva de estos pacientes está siempre preservada ${ }^{4}$. Su causa es una mutación homocigota, en el gen de sobrevida de la motoneurona, $S M N$, ubicado en el brazo largo del cromosoma 5 (5q11.1-13.3). Este gen está presente en múltiples copias en el genoma humano, una telomérica $S M N 1$ y varias copias centroméricas, SMN2 que se diferencian en sólo cinco nucleótidos. El gen SMN2 presenta una tendencia a un ensamblaje génico alternativo (en inglés, "alternative splicing") durante la transcripción del ARNm que origina una proteína truncada, con sólo $10 \%$ de la proteína SMN completa. Esta proteína normal no logra compensar la pérdida de la proteína por mutación del gen $S M N 1^{3,5}$. Deleciones del exón 7 y 8 o sólo del 7 del gen SMN1 son responsables de más de $95 \%$ de los casos de $\mathrm{AME}^{6}$. De este modo, la detección de una deleción homocigota de al menos el exón 7 de $S M N 1$, constituye una herramienta para el diagnóstico de AME que alcanza una sensibilidad cercana a $95 \%$ y una especificidad de $99 \%{ }^{6}$. Los diversos subtipos clínicos de AME se han clasificado según la edad de aparición de los síntomas y su evolución ${ }^{2}$ y se muestran en la Tabla 1.

El objetivo de este trabajo es presentar las características clínicas y neuro-fisiológicas de pacientes chilenos con AME y los resultados de la confirmación genética molecular tras la implementación del test diagnóstico genético-molecular en nuestro país.

\section{Pacientes y Método}

Estudio de una cohorte multicéntrica, descriptivo, retrospectivo a partir de los registros clínicos de pacientes con diagnóstico y confirmación genética de AME evaluados entre enero de 2003 y abril de 2010. Todos los pacientes fueron clínicamente evaluados y seguidos por los autores. Se clasificó a los pacientes en los diferentes tipos de AME de acuerdo a la Tabla 1 . Se consignó manifestación clínica inicial, edad del diagnóstico clínico, presencia /ausencia de temblor lingual, temblor de manos (poliminimioclonus), escoliosis, edad de pérdida de la marcha y edad de fallecimiento. Se analizó el estudio neurofisiológico y biopsia muscular cuando procedió.

El estudio genético molecular fue realizado en el extranjero hasta el año 2005. Posteriormente, se implementó la técnica en el laboratorio de Anatomía Patológica de Clínica Las Condes.

\section{Implementación de la detección genético molecular de la AME}

El estudio molecular se realizó a partir del ácido deoxirribonucleico (ADN) extraído de linfocitos en sangre periférica de pacientes con sospecha diagnóstica de AME mediante la técnica de reacción de polimerasa en cadena (PCR) con amplificación de los exones 7 y 8 del gen $S M N 1$. Dado que los partidores de la reacción amplifican indistintamente ambas versiones del gen SMN1 y $S M N 2$, estos debieron diferenciarse por el análisis de polimorfismo de longitud de fragmentos de restricción (RFLP) ${ }^{5}$. Los productos de amplificación y los fragmentos de restricción fueron visua-

Tabla 1. Clasificación de los subtipos clínicos de atrofia muscular espinal según edad de inicio de síntomas y gravedad de evolución

\begin{tabular}{|lccc|}
\hline Tipo & \multicolumn{1}{c}{ Inicio } & Maxima habilidad & Fallecimiento \\
$\begin{array}{l}\text { Tipo I } \\
\text { Enfermedad de Werdnig-Hoffmann }\end{array}$ & $<6$ meses & No logra sentarse & $<2$ años \\
$\begin{array}{l}\text { Tipo II } \\
\text { Intermedio }\end{array}$ & $7-18$ meses & No logra caminar & $>2$ años \\
$\begin{array}{l}\text { Tipo III } \\
\text { Enfermedad de Kugelberg-Welander }\end{array}$ & $>18$ meses & Marcha autónoma & Adulto \\
$\begin{array}{l}\text { Tipo IV } \\
\text { 2a }\end{array}$ & $2^{\text {a }}$ década & $\begin{array}{l}\text { Marcha autónoma } \\
\text { Vida adulta }\end{array}$ & Adulto \\
\hline
\end{tabular}




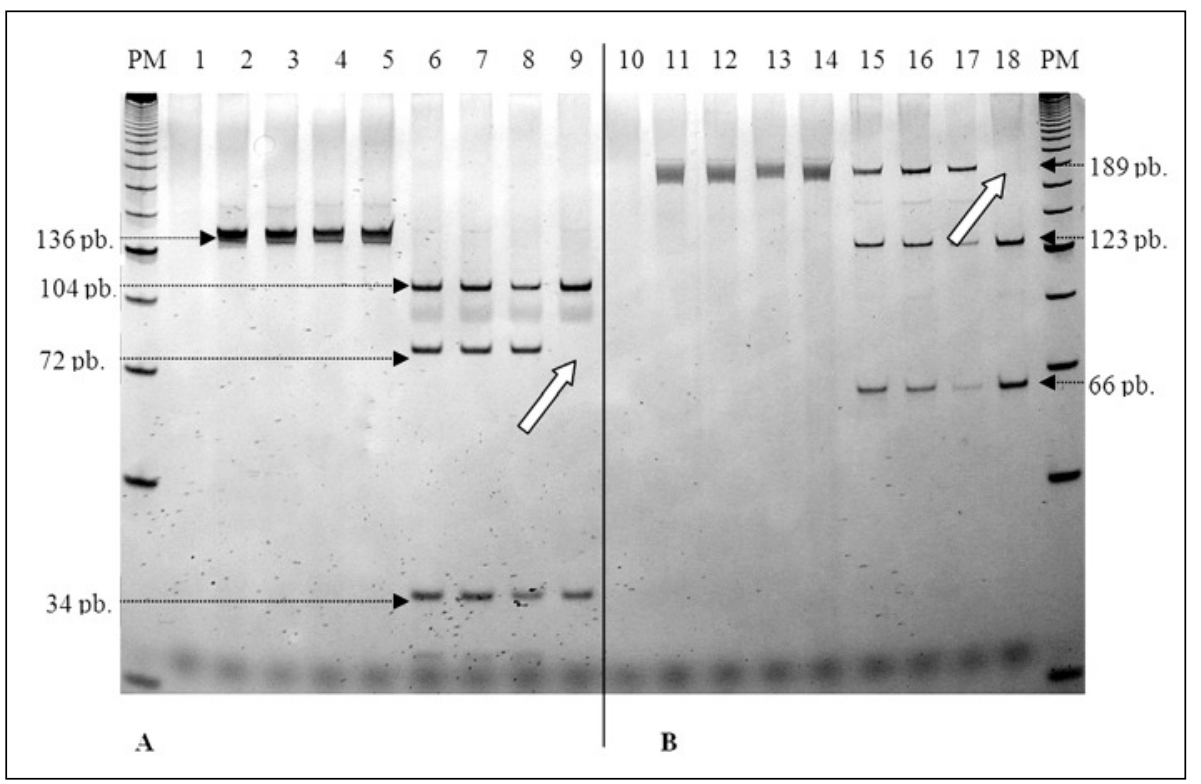

Figura 1.

lizados en geles de acrilamida al $8 \%$ y revelados con tinción de plata. Se interpretó como deleción homocigota del exón 7 del gen SMN1 la falta de 1 de los tres fragmentos de restricción (Figura 1A). La deleción homocigota del exón 8 del mismo gen, se evidenció por la ausencia de la banda de 189 pares de base sin digerir del gen $S M N 1$, junto con la presencia de los dos fragmentos de restricción del gen SMN2 (Figura 1B). Se realizaron en conjunto con los exámenes, controles positivos y negativos usando ADN caracterizado previamente en el Instituto de Genética Humana de la Universidad de Bonn, Alemania.

\section{Resultados}

La serie está compuesta por 26 pacientes; 15 (58\%), de sexo masculino, con edades en el momento del diagnóstico que fluctuaron entre 1 mes y 18 años. Cuatro $(15,4 \%)$ pacientes tenían los criterios descritos de AME tipo I, 11 (42,3\%) de AME tipo II y $11(42,3 \%)$ de AME tipo III. Esta serie de población pediátrica no contempla pacientes con la forma tipo IV de la enfermedad.

\section{Características clínicas}

Los 4 pacientes con AME tipo I se presentaron con escasos movimientos espontáneos, hipotonía y debilidad muscular. Presentaban una hipotonía generalizada de predominio proximal con las caderas en abducción, sin movimientos contra la gravedad, lo que contrastaba con la agudeza y vivacidad de la mirada y de los movimientos de la cara. Destacaba la presencia de fasciculaciones linguales en todos ellos y en dos, la presencia de trastornos de la deglución en el curso de su evolución. El intervalo entre la consulta y la sospecha diagnóstica fluctuó entre 1 y 3 meses; en todos ellos el diagnóstico se confirmó mediante el estudio de la deleción del gen SMN1. Estos 4 pacientes fallecieron de insuficiencia respiratoria entre los 8 y 14 meses de edad.

Las Tablas 2 y 3 resumen las características clínicas y los resultados del estudio genético molecular de los pacientes con AME tipo II y tipo III respectivamente.

Todos los pacientes con AME tipo II consultaron por algún grado de retraso en el desarrollo motor, destacando la imposibilidad en mantenerse de pie sin apoyo y en sostener su peso. El diagnóstico se estableció entre los 10 y 23 meses de edad. En aquellos pacientes con sospecha clínica más precoz, ésta se realizó por la presencia de una importante hipotonía e hiperlaxitud, junto al antecedente de retraso en la adquisición de control cefálico y sedestación. Ninguno logró una marcha autónoma. Seis $(54,5 \%)$ de los 11 pacientes 
se presentaron con escoliosis en el momento del diagnóstico, 4 de ellos ya sometidos a cirugía ortopédica de la columna. Todos están vivos a la fecha y los dos pacientes de mayor edad han realizado estudios superiores.

En 7 de 11 pacientes con AME tipo III (que son aquellos que lograron la marcha autónoma), el diagnóstico se realizó entre los 18 y 36 meses de edad. Ellos presentaban caídas frecuentes, dificultades para pararse del suelo, marcha bamboleante y el temblor característico de sus manos, denominado poliminimioclonus. En 4 de 11, el diagnóstico fue más tardío; de éstos, 2 pacientes tenían diagnóstico previo en otros centros de

Tabla 2. Características clínicas y estudio molecular de pacientes con atrofia muscular espinal tipo II

\begin{tabular}{|ccclccc|}
\hline $\mathbf{n}$ & Sexo & $\begin{array}{c}\text { Edad } \\
\text { diagnóstico } \\
\text { (meses) }\end{array}$ & \multicolumn{1}{c}{ Síntoma inicial } & $\begin{array}{c}\text { Polimini- } \\
\text { mioclono }\end{array}$ & $\begin{array}{c}\text { Escoliosis } \\
\text { Deleción } \\
\text { SMN1 }\end{array}$ \\
\hline 1 & $\mathrm{M}$ & 18 & Hipotonía retraso sedestación & Sí & No & Exón 7-8 \\
\hline 2 & $\mathrm{M}$ & 13 & Imposibilidad de mantenerse de pie & Sí & No & Exón 7-8 \\
\hline 3 & $\mathrm{M}$ & 10 & Hipotonía retraso en control cefálico & Sí & Sí & Exón 7-8 \\
\hline 4 & $\mathrm{M}$ & 10 & Retraso motor & No & Sí & Exón 7-8 \\
\hline 5 & $\mathrm{~F}$ & 12 & Retraso motor & No & Sí & Exón 7-8 \\
6 & $\mathrm{M}$ & 23 & Dificultades para moverse & Sí & Sí & Exón 7-8 \\
\hline 7 & $\mathrm{M}$ & 14 & Imposibilidad de mantenerse de pie & No & Sí & Exón 7-8 \\
\hline 8 & $\mathrm{~F}$ & 12 & Imposibilidad de mantenerse de pie & Sí & No & Exón 7-8 \\
\hline 9 & $\mathrm{~F}$ & 16 & Imposibilidad de mantenerse de pie & Sí & Si & Exón 7-8 \\
\hline 10 & $\mathrm{M}$ & 15 & Imposibilidad de mantenerse de pie & Sí & No & Exón 7 \\
\hline 11 & $\mathrm{M}$ & 19 & Imposibilidad de mantenerse de pie & Sí & No & Exón 7-8 \\
\hline
\end{tabular}

Tabla 3. Características clínicas y estudio molecular de pacientes con atrofia muscular espinal tipo III

\begin{tabular}{|c|c|c|c|c|c|c|c|}
\hline $\mathbf{n}$ & Sexo & $\begin{array}{c}\text { Edad } \\
\text { diagnóstico } \\
\text { (años) }\end{array}$ & Síntoma inicial & $\begin{array}{c}{ }^{* C K} \\
\text { total } \\
\text { U/L }\end{array}$ & $\begin{array}{l}\text { Polimini- } \\
\text { mioclono }\end{array}$ & Escoliosis & $\begin{array}{l}\text { Deleción } \\
\text { SMN1 }\end{array}$ \\
\hline 1 & M & 4 & $\begin{array}{l}\text { Marcha anadina dificultad para } \\
\text { pararse del suelo }\end{array}$ & 80 & No & No & Exón 7-8 \\
\hline 2 & M & 18 & Marcha con debilidad & 191 & Sí & No & Exón 7 \\
\hline 3 & $\mathrm{~F}$ & 6 & Caídas frecuentes & 170 & Sí & Sí & Exón 7-8 \\
\hline 4 & $\mathrm{~F}$ & 2 & Dificultades para parase del suelo & 70 & Sí & No & Exón 7 \\
\hline 5 & M & 2 & Caídas frecuentes & 166 & Sí & Sí & Exón 7 \\
\hline 6 & M & 1,5 & Retraso en desarrollo motor & 139 & Sí & No & Exón 7-8 \\
\hline 7 & M & 3 & Dificultades para caminar & ND & Sí & Sí & Exón 7-8 \\
\hline 8 & $\mathrm{~F}$ & 2 & Pierde marcha posterior a infección & ND & Sí & Sí & Exón 7-8 \\
\hline 9 & $\mathrm{~F}$ & 3 & Dificultad para subir escaleras & ND & Sí & No & Exón 7-8 \\
\hline 10 & M & 2 & Dificultades para correr & 130 & Sí & Sí & Exón 7-8 \\
\hline 11 & M & 13 & Caídas frecuentes & 434 & Sí & Sí & Exón 7-8 \\
\hline
\end{tabular}

${ }^{*}$ CK valor normal < de 180 U/L ; ND: No determinado. 
distrofia muscular de las cinturas, con un fenotipo caracterizado por debilidad de las cinturas pélvica y escapular de evolución lentamente progresiva con importante amiotrofia generalizada, muy marcada en los cuadriceps. En otro, la presencia de una hipertrofia de pantorrillas asociada a debilidad de cintura pélvica fue interpretada inicialmente como una probable distrofia muscular tipo Duchenne. El restante, fue un paciente procedente de Perú sin diagnóstico previo. En ellos, el examen genético realizó el diagnóstico definitivo de AME.

\section{Estudio electrofisiológico}

En todos los pacientes con AME tipo I y II, el estudio electromiográfico mostró signos de denervación, con ondas agudas positivas y fibrilaciones acompañados de signos de re-inervación crónica con potenciales de unidad motora de amplitud y duración acrecentadas y reclutamiento disminuido. En los pacientes con AME tipo III se observaron de preferencia signos de re-inervación crónica marcada con potenciales de unidad motora de gran amplitud, característica que orientó el diagnóstico en aquellos pacientes con sospecha inicial de distrofia muscular de las cinturas. El estudio de neuroconducción motora y sensitiva fue normal en todos los pacientes con AME tipo II y III. En los pacientes con AME tipo I, la amplitud de los potenciales motores estaba disminuida.

\section{Biopsia Muscular}

A $9(34,6 \%)$ pacientes se les realizó una biopsia muscular; 7 de ellos fueron pacientes con AME tipo III. En 8, las alteraciones encontradas permitieron orientar el diagnóstico, observándose el característico agrupamiento de fibras de la atrofia neurogénica (Figura 2). En un paciente la biopsia muscular se informó como compatible con una distrofia muscular. No se tuvo acceso a ella para re-evaluarla.

\section{Estudio genético molecular}

De los 26 pacientes evaluados, $22(84,6 \%)$ presentaron una deleción homocigota de los exones 7 y 8 del gen $S M N 1$. En los otros 4 sólo se encontró una deleción del exón 7 del gen SMN1. Podemos agregar que en otros 17 pacientes que tenían la sospecha clínica de AME, no se encontró ninguna de estas 2 deleciones haciendo muy improbable este diagnóstico.

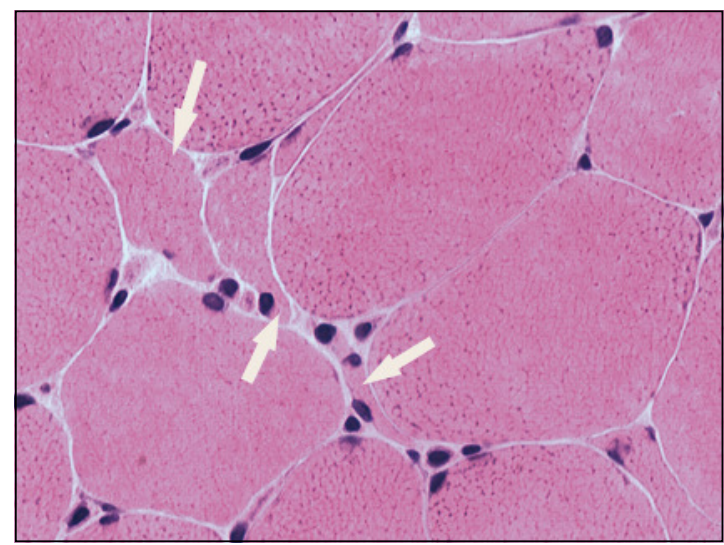

Figura 2.

\section{Discusión}

En 1891, Guido Werdnig, neurólogo austríaco, describió por primera vez las características clínicas de dos pacientes afectados con una atrofia muscular progresiva. Dos años más tarde, Johann Hoffmann, neurólogo alemán, estableció el origen espinal de la enfermedad al demostrar en autopsias la degeneración de las neuronas del asta anterior de la médula espinal ${ }^{7}$. En 1954 y 1956, Eric Kugelberg y Lisa Welander en Suecia, describieron la primera serie de una forma de inicio tardío posterior a la edad de adquisición de la marcha, de evolución lenta que pasó a denominarse enfermedad de Kugelberg-Welander ${ }^{7,8}$. Sólo en 1990, los grupos de investigadores dirigidos por Judit Melki en Francia y Conrad Gilliam en los Estados Unidos de Norteamérica, localizaron el gen anómalo responsable de todas las formas de la AME, en el brazo largo del cromosoma 5 (5q11.2-q13.3) $)^{9,10}$. Cinco años más tarde Lefebre y Melki identificaron y caracterizaron el gen SMN1, cuya deleción origina la enfermedad ${ }^{11}$. Los primeros estudios que sugirieron las bases moleculares que determinan la gravedad de la enfermedad, fue la observación en 1997 de una estrecha correlación inversa entre la cantidad de proteína SMN codificada por el gen $S M N 2$ y la gravedad del fenotipo clínico ${ }^{12,13}$. El número de copias del gen SMN2 se correlaciona directamente con el tipo de enfermedad y sobrevida $^{14,15}$. El 80\% de los pacientes con AME tipo 1 muestran sólo una o dos copias del gen $S M N 2^{15}$.

Esta cohorte de pacientes chilenos con AME ejemplifica el amplio espectro clínico y fenotípico 
de esta enfermedad y pone de manifiesto la mayor dificultad diagnóstica en aquellos casos menos graves clasificados como AME tipo III. Éstos, se pueden confundir con distrofias musculares y explica el predominio de biopsias musculares realizadas en nuestros pacientes con AME tipo III. Incluso, ha sido descrito en casos de hermanos con la misma deleción genética del gen $S M N 1$, formas fenotípicas diferentes; uno de los hermanos con una presentación clásica de AME tipo III y el otro con un fenotipo de distrofia muscular (16). Nicole $S$ y coautores recuerdan publicaciones de la era pre-genética de la enfermedad en que se describía que cerca de un cuarto de los pacientes con diagnóstico clínico de AME tipo III se presentaban con un fenotipo distrófico con aumento de los niveles sanguíneos de creatinkinasa (CK) e histopatología miopática ${ }^{17}$.

Actualmente, acceder al estudio genético molecular permite la certificación del diagnóstico, proponer una conducta terapéutica y otorgar un pronóstico y consejo genético apropiado. A los pacientes con una presentación clínica sugerente de una AME debe solicitársele el estudio de la deleción del gen SMN1 que tiene una especificidad cercana al $100 \%{ }^{3}$. Antes de la disponibilidad del estudio genético, el diagnóstico de AME necesitaba frecuentemente de la realización de una biopsia muscular. En nuestra serie, 9 pacientes requirieron de ella porque la sospecha clínica se realizó antes de la implementación del test genético o hubo dificultad en acceder al estudio en el extranjero y en otros dos, debido a que el diagnóstico clínico orientaba a otro tipo de enfermedad. Hoy en día, con la disponibilidad de la confirmación genética de AME, la biopsia muscular puede ser evitada.

El predominio en esta serie de formas tipo II y tipo III de AME, pese a que el tipo I representa más del $50 \%$ de los afectados en la literatura internacional $^{2}$, puede explicarse porque los pacientes con enfermedad de Werdnig Hoffmann (AME tipo I), de pronóstico más sombrío, fallezcan precozmente de complicaciones respiratorias quizás sin un diagnóstico de certeza.

En nuestros pacientes con AME tipo II se menciona una escoliosis significativa en el 54,5\% de ellos, cuatro ya operados. De acuerdo a Sucato todos los niños con una AME tipo I y II desarrollarán diferentes grados de escoliosis de curso progresivo de inicio más precoz mientras mayor sea el grado de debilidad de la enfermedad ${ }^{18}$. Esta complicación contribuye a ensombrecer el pronóstico funcional respiratorio, además de ocasionar importante dolor, de manera que un tratamiento quirúrgico oportuno mejora la calidad de vida y pronóstico vital de este grupo de pacientes.

No existe hoy tratamiento curativo para esta enfermedad, sin embargo, los avances en la comprensión de su patogénesis han contribuido al desarrollo de ensayos terapéuticos específicos orientados a incrementar la producción de la proteína funcional completa $\mathrm{SMN}$ a partir del gen $S M N 2$. Estos ensayos se han realizado con ácido valproico, fenilbutirato de sodio e hidroxiurea ${ }^{19-21}$. Específicamente las estrategias se han enfocado a aumentar la inclusión del exón 7 en el trascripto de RNA mensajero del gen $S M N 2^{19-21}$, a la sobre regulación de la trascripción de $S M N 2$ por activación del promotor ${ }^{22}$, a la modulación de la trascripción de la proteína $S M N 2^{23}$ y a la prevención de la degradación de la proteína $\mathrm{SMN}^{24}$.

En la práctica clínica su pronóstico a corto y mediano plazo depende de la precocidad de la implementación de un tratamiento nutricional, respiratorio, ortopédico, fisioterápico y neurológico en forma coordinada y permanente en el tiempo. En este sentido la declaración de consenso del año 2007 para el estándar de cuidados en atrofia muscular espinal es una guía práctica para el manejo clínico de pacientes con AME ${ }^{25,26}$. (Tabla 4). No debemos desconocer que existen publicaciones con sobrevida prolongada de pacientes con AME tipo I (enfermedad de Werdnig Hoffmann) por sobre los 10 años de edad, con una adecuada implementación de protocolos de ventilación no invasiva $^{27,28}$. En ocasiones, los sistemas de ayuda social y de rehabilitación presentan carencias para un tratamiento óptimo de estos pacientes de suyos complejos.

Si bien es cierto que, la presente serie es sólo un estudio retrospectivo con un limitado número de casos, lo que le resta fuerza a sus implicancias, pensamos que la certeza y oportunidad del diagnóstico de AME, podría conllevar a una considerable mejoría en la sobrevida y calidad de vida de estos enfermos. Así, la caracterización clínica, electrofisiológica y confirmación genética que aquí presentamos, constituye un punto de partida, para el mejor tratamiento de estos pacientes. Destacamos aquí la implementación en Chile del estudio genético molecular, lo que constituye un aporte en los cuidados de estos niños en nuestro medio. 


\section{Tabla 4.Rcomendaciones de la declaración de consenso para el estándar de cuidados en atrofia muscular espinal ${ }^{25}$}

\section{Recomendaciones para pacientes con diagnóstico reciente de AME}

- Confirmar el diagnóstico genético con el estudio del gen SMN-1

- Clasificar AME en tipos I, II, III, o IV de acuerdo al nivel más alto de función motora alcanzada (ej. no se sienta, se sienta, camina)

- Proporcionar referencias para un cuidado multidisciplinario

- Proporcionar información de grupos de apoyo de AME y ensayos clínicos terapéuticos

- Ofrecer consejo genético

\section{Cuidados bronco-pulmonares}

- Evaluar el compromiso respiratorio: tos, efectividad de la tos, trastornos respiratorios con el sueño, hipercapnia nocturna, infecciones recurrentes, hipercapnia diurna

- Asistencia para mantener vía aérea despejada: asistencia a la tos, movilización de secreciones, aspiración

- Considerar ventilación no invasiva (BIPAP) o invasiva (intubación, traqueostomía)

- Ofrecer tratamiento agudo: normalización del intercambio gaseoso, antibióticos, soporte nutricional, tratamiento del reflujo gastro-esofágico

- Establecer objetivos del tratamiento: maximizar calidad de vida, confort y sobrevida a largo plazo

\section{Cuidados gastrointestinales y nutricionales}

- Seguimiento de la velocidad de crecimiento, peso, relación peso/talla

- Fraccionar la alimentación para evitar fatigas y tiempos de alimentación prolongados

- Investigar problemas de deglución: video fluoroscopia, estudios de deglución

- Indicar proactivamente apoyo nutricional: implementar gastrostomía o sonda de alimentación naso-gástrica o nasoyeyunal cuando se reconozca una inadecuada ingesta oral

- Minimizar el reflujo e gastroesofágico (asociado a neumonías aspirativas y eventos con riesgos de vida) con una gastrostomía más fundoplicatura de Nissen

\section{Cuidados ortopédicos y de rehabilitación}

- Reconocer problemas: contracturas, deformidades de columna, disminución de movilidad, osteopenia, fracturas.

- Evaluar rangos de movimiento, fuerza, y función motora usando escalas validadas, objetivas

- Usar asistencia técnica: sillas de rueda para la movilización, bipedestadores, órtesis de extremidades y corporales

- Prescribir tratamiento kinésico y de terapia ocupacional en forma regular

- Corrección quirúrgica de deformidades esqueléticas que afecten la función respiratoria o la deambulación

\section{Cuidados paliativos}

- Considerar balance entre prolongar la vida y calidad de vida

- Presentar todas las opciones de tratamiento de manera abierta transparente y equilibrada a todos los que toman decisiones

- Discutir precozmente los planes para el tratamiento en situaciones de emergencia

- Ofrecer ventilación no invasiva y gastrostomía que pueden reducir la necesidad de un apoyo emergente posterior

- Discutir medidas terminales, especialmente tratamiento del dolor

Adaptado de Curr Treat Options Neurol. 2008 ref Nº 25.

Agradecimientos: A la Dra. Prof. Dr. Brunhilde Wirth del Instituto de Genética Humana de la Universidad de Bonn, Alemania, por facilitar muestras de ADN controles; al Dr. Roger Gerjman del Departamento de Anatomía Patológica de la Pontificia Universidad Católica de Chile, por facilitar la microfotografía de uno de nuestros pacientes y al Dr. Juan Pablo Torres, sub-director académico de Clínica Las Condes, por sus valiosas sugerencias y lectura crítica del manuscrito.

\section{Referencias}

1. Monani UR. Spinal Muscular Atrophy: A Deficiency in a Ubiquitous Protein; a Motor Neuron-Specific Disease. Neuron 2005; 48: 885-96.

2. Lunn MR, Wang $\mathrm{CH}$. Spinal muscular atrophy. Lancet 2008; 371: 2120-33.

3. Burlet P, Bürglen L, Clermont O, Lefebvre S, Viollet L, Munnich A, Melki J. Large scale deletions of the $5 \mathrm{q} 13$ region are specific to Werdnig-Hoffmann disease. J Med 
Genet 1996; 33: 281-3.

4. Von Gontard A, Zerres K, Backes M, LaufersweilerPlass C, Wendland C, Melchers P, et al. Intelligence and cognitive functions in children and adolescents with spinal muscular atrophy. Neuromuscul Disord 2002; 12: $130-6$

5. Scheffer H, Cobben Jm, Matthijs G, Wirth B. Best practice guidelines for molecular analysis in spinal muscular atrophy. Eur J Hum.Genet.2001; 9: 484-91.

6. Ogino S, Wilson R. Spinal muscular atrophy: molecular genetics and diagnostics. Expert Re Mol Diagn 2004; 4: 15-29.

7. Dubowitz V. Ramblings in the history of spinal muscular atrophy. Neuromuscul. Disord 2009; 69-73.

8. Borg K, Joélius B. Lisa Welander and Eric Kugelberg-two Swedish myologists in the footsteps of Edward Meryon. Neuromuscul Disord 2004; 14: 383-6.

9. Melki J, Sheth P, Abdelhak S, Burlet P, Bachelot MF, Lathrop MG, et al. Mapping of acute (type I) spinal muscular atrophy to chromosome 5q12-q14. Lancet 1990; 336: 271-3.

10. Gilliam TC, Brzustowicz LM, Castilla LH, Lehner T, Penchaszadeh GK, Daniels RJ, et al. Genetic homogeneity between acute and chronic forms of spinal muscular atrophy. Nature 1990; 28; 345: 823-5.

11. Lefebvre S, Bürglen L, Reboullet S, Clermont O, Burlet $\mathrm{P}$, Viollet L, et al. Identification and characterization of a spinal muscular atrophy-determining gene. Cell. 1995; 80: 155-65.

12. Lefebvre S, Burlet P, Liu Q, Bertrandy S, Clermont O, Munnich A, et al. Correlation between severity and SMN protein level in spinal muscular atrophy. Nat Genet 1997; 16: 265-9.

13. Coovert D, Le T, Mcandrew P, Strasswimmer J, Crawford T, Mendell JR, et al. The survival motor neuron protein in spinal muscular atrophy. Hum Mol Genet 1997; 6: 1205-14.

14. Crawford TO. Spinal Muscular Atrophies. En Jones HR, De Vivo DC, Darras BT editores, Neuromuscular Disorders of Infancy, Childhood and Adolescence: A Clinician's Approach. Edit: Butterworth Heinemann, Philadephia, PA, USA, 2003; p. 145-66.

15. Feldkotter M, Schwarzer V, Wirth R, Wienker TF, Wirth B. Quantitative analyses of SMN1 and SMN2 based on real-time light Cycler PCR: fast and highly reliable carrier testing and prediction of severity of spinal muscular atrophy. Am J Hum Genet 2002; 70: 358-68.

16. Muqit MM, Moss J, Sewry C, Lane RJ. Phenotypic varia- bility in siblings with type III spinal muscular atrophy. J Neur Neurosur Psy 2004; 75: 1762-4.

17. Nicole S, Cifuentes-Díaz C, Frugier T, Melki J. Spinal muscular atrophy: recent advances and future prospects. Muscle Nerve 2002; 26: 4-13.

18. Sucato DJ. Spine deformity in spinal muscular atrophy. J Bone Joint Surg Am 2007; 89: 148-54.

19. Brichta L, Hofmann Y, Hahnen E, Siebzehnrubl FA, Raschke H, Blumcke, et al. Valproic acid increases the SMN2 protein level: a well-known drug as a potential therapy for spinal muscular atrophy. Human Molecular Genetics 2003; 12: 2481-9.

20. Chang JG, Hsieh-Li HM, Jong YJ, Wang NM, Tsai CH, $\mathrm{Li} \mathrm{H}$. Treatment of spinal muscular atrophy by sodium butyrate. PNAS 2001; 98: 9808-13.

21. Grzeschik SM, Ganta M, Prior TW, Heavlin WD, Wang $\mathrm{CH}$. Hydroxyurea enhances SMN2 gene expression in spinal muscular atrophy cells. Ann Neurol 2005; 58: 194-202.

22. Jarecki J, Chen X, Bernardino A, Coovert DD, Whitney $\mathrm{M}$, Burghes A, et al. Diverse small-molecule modulators of SMN expression found by high-throughput compound screening: early leads towards a therapeutic for spinal muscular atrophy. Hum Mol Genet 2005; 14: 2003-18.

23. Wolstencroft EC, Mattis V, Bajer AA, Young PJ, Lorson CL. A non-sequence-specifi c requirement for SMN protein activity: the role of aminoglycosides in inducing elevated SMN protein levels. Hum Mol Genet 2005; 14: 1199-210.

24. Waza M, Adachi H, Katsuno M, Minamiyama M, Tanaka F, Sobue G. Alleviating neurodegeneration by an anticancer agent: an Hsp90 inhibitor (17-AAG). Ann N Y Acad Sci 2006; 1086: 21-34.

25. Wang $\mathrm{CH}$, Lunn MR. Spinal muscular atrophy: advances in research and consensus on care of patients. Curr Treat Options Neurol 2008; 10: 420-8.

26. Wang CH, Finkel RS, Bertini ES, Schroth M, Simonds A, Wong B, et al. Consensus statement for standard of care in spinal muscular atrophy. J Child Neurol 2007; 22: 1027-49.

27. Bach JR, Saltstein K, Sinquee D, Weaver B, Komaroff E. Long-term survival in Werdnig-Hoffmann disease. Am J Phys Med Rehabil 2007; 86: 339-45.

28. Borkowska J, Rudnik-Schoneborn S, HausmanowaPetrusewicz I, Zerres K. Early infantile form of spinal muscular atrophy (Werdnig-Hoffmann disease) with prolonged survival. Folia Neuropathol 2002; 40: 19-26. 